\title{
Comparison of nasal and tracheobronchial clearance by similar techniques in normal subjects
}

\author{
A B MILlAR, J E AGNEW, S P NEWMAN, M T LOPEZ-VIDRIERO, D PAVIA, \\ S W CLARKE

\begin{abstract}
From the Departments of Thoracic Medicine and Medical Physics, Royal Free Hospital and Medical School, London
\end{abstract}

\begin{abstract}
Nasal and tracheobronchial mucociliary clearance have been compared in 10 healthy subjects. Nasal clearance was measured by monitoring the rate of removal of $2 \mu \mathrm{m}$ diameter Teflon particles, labelled with ${ }^{99 \mathrm{~m}} \mathrm{Tc}$, which had been placed in the anterior part of the nose. Tracheobronchial clearance was measured with an objective radioaerosol technique, $5 \mu \mathrm{m}$ diameter polystyrene particles being used. With these comparable techniques there was a close correlation between the nasal mucociliary clearance rate and both the area under the tracheobronchial clearance curve from 0 to 6 hours after radioaerosol inhalation $\left(r_{s}=-0.94, p<0.001\right)$ and the area under the tracheobronchial clearance curve from 0 to 2.5 hours after inhalation $\left(r_{s}=-0.79\right.$, $\mathrm{p}<0.01)$. The rate of clearance of small particles from the nose may thus be a useful guide to tracheobronchial clearance in healthy individuals.
\end{abstract}

Nasal mucociliary clearance has an important role as a defence mechanism for the upper airway. ${ }^{1-3}$ The nose is an easily accessible organ and so in many centres the measurement of nasal mucociliary clearance has been used as a screening procedure for congenital abnormalities that would alter mucociliary clearance in the nose and lungs. The relationship between nasal and tracheobronchial clearance is not, however, clear. Previous studies ${ }^{45}$ comparing the two have reported diverse results. This may be due to the different techniques used. In the present study we have used broadly similar techniques for the two measurements and so consider that we can determine the interrelationship between nasal and tracheobronchial clearance in normal subjects.

\section{Methods}

\section{TECHNIQUE}

Nasal clearance was measured with radiolabelled particles in a technique modified from that of Black $e t$ $a l .{ }^{6}$ Teflon particles (physical diameter $2 \mu \mathrm{m}$, aerodynamic diameter $3.2 \mu \mathrm{m}$ ) labelled with the radionuclide technetium $99 \mathrm{~m}\left({ }^{99 \mathrm{~m}} \mathrm{Tc}\right)$ were produced by a spinning top generator, ${ }^{7}$ and were collected and then

Address for reprint requests: Dr D Pavia, Department of Thoracic Medicine, Royal Free Hospital, London NW3 2QG.

Accepted 22 April 1986 washed twice in $40 \%$ ethanol before being suspended in $0.2 \mathrm{ml}$ of $40 \%$ ethanol. ${ }^{8}$ The subject's nose was examined and the inferior turbinate visualised, an auriscope being used as a speculum. The apparatus for the administration of the particle suspension consisted of a piece of narrow plastic tubing attached to a brass $\mathrm{T}$ piece. The latter was connected via a solenoid operated valve to a reservoir of compressed air at $7 \mathrm{kPa}$. Then $2 \mu \mathrm{l}$ of the particle suspension containing $370 \mathrm{kBq}(10 \mu \mathrm{Ci}){ }^{99 \mathrm{~m}} \mathrm{Tc}$ were drawn into a plastic pipette tip with an automatic pipette. This pipette tip was attached on to the plastic tubing and over it a second pipette tip was placed to prevent any movement of the suspension by touching nasal hairs. The pipette tips were introduced through the auriscope and the suspension was blown via the operation of the solenoid valve on to the inferior turbinate of the right nostril.

The subject was positioned immediately with his or her head against a large field of view gamma camera (General Electric), which was connected on line to a Varian V-77 600 computer. Lateral views of the nasal region were taken at one minute intervals for 20 minutes and stored on magnetic tape. Each subject had radioactive markers (labelled with cobalt 57) on the chin, the nape of the neck and the forehead throughout the study for anatomical reference purposes. By using these fixed reference points a superimposed grid was placed identically on a computer 


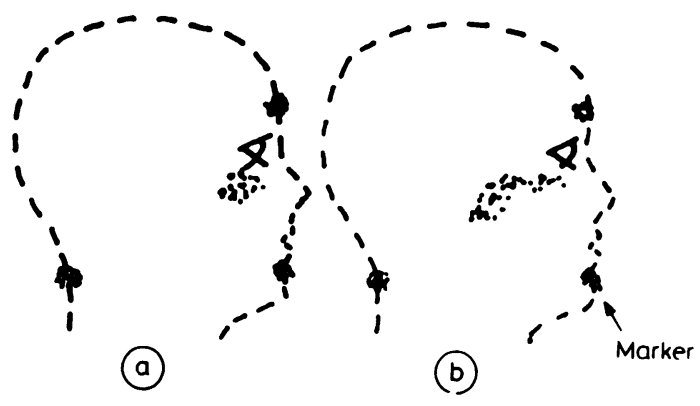

Fig 1 Diagrammatic representation of computer printout showing nasal deposition pattern in relation to fixed markers: (a) initial deposition; (b) after 15 minutes.

print out of each view as depicted in fig 1. The XY coordinates of the radioactive particle bolus were calculated from the fixed ${ }^{57} \mathrm{Co}$ markers for each view. Thus a graph of position of this bolus as a function of time was produced. The real chin to nape of neck measurement was compared with that on the printouts to give scale so that nasal clearance could be calculated in $\mathrm{cm} / \mathrm{min}$. Scans of the chest showed that no radioactive particles reached the lungs during the administrative procedures.

To assess the reproducibility of this technique we repeated the measurement of nasal clearance at an interval of 6-12 months after the initial studies.

On a separate day a non-invasive objective radioaerosol technique, described in detail previously, ${ }^{9}$ was used to measure lung mucociliary clearance. Polystyrene particles of $5 \mu \mathrm{m}$ diameter, again labelled with ${ }^{99 \mathrm{~m}} \mathrm{Tc}$, were produced inside an airtight tank by a spinning top generator. ${ }^{7}$ The subjects, wearing a nose clip, inhaled the radioaerosol by taking eight single breaths via a mouthpiece from functional residual capacity to an automatically preset volume of $450 \mathrm{ml}$, followed by a three second breath hold before they exhaled. Oral rinsing and swallowing of water removed particles from the mouth and oesophagus. The amount of initially deposited aerosol within the lungs and its subsequent clearance were monitored by two collimated scintillation counters located anteriorly and posteriorly to the chest. Measurements were made at hourly intervals for six hours after inhalation of the radioaerosol and one final count was made at 24 hours-which was taken to be an estimate of alveolar deposition. ${ }^{10}$ Count corrections were made for background radiation and physical decay of the radionuclide ( $T^{1 / 2} 6$ hours). The estimate of alveolar deposition was subtracted from the whole lung clearance curve to obtain the curve of tracheobronchial clearance. None of the subjects coughed during the six hours' observation period. The areas
Millar, Agnew, Newman, Lopez-Vidriero, Pavia, Clarke

under the tracheobronchial clearance curve for both 0-2.5 hours and 0-6 hours were calculated for each subject. The area under the tracheobronchial clea ance curve is representative of the mean rate of cleas ance and inversely proportional to it. The first 28 hour period represents clearance from the more prots imal airways and the 6 hour period includes clearance from virtually all the ciliated airways.

\section{SUBJECTS}

Ten volunteer subjects were recruited and gave informed consent to the procedures. Nine were nori smokers and one an ex-smoker.

None of the subjects had had an upper respiratory tract infection in the two months before assessmene् The group comprised six men and four women-응 mean (SEM) age $28(2)$ years, height $1.70(0.04) \mathrm{m}$ and weight $65(3) \mathrm{kg}$. Their pulmonary function indio ces, expressed as percentages of predicted value were: FEV 120 (5), forced vital capacity (FVC) $11 \%$ (4), peak expiratory flow (PEF) 104 (4) (normat values are taken from Cotes (11)).

\section{Results}

The mean nasal clearance rate was $1.016 \mathrm{~cm} / \mathrm{min}$ witlo a range of $0.5-1.7 \mathrm{~cm} / \mathrm{min}$. When the nasal clearance measurements were repeated 6-12 months after the initial studies the measurements were reproduced to within $\pm 11 \%$. The mean (SEM) area under the tracheobronchial clearance curve for 6 hours was $21 \theta$ (22) arbitrary units (range 103.5-319.8) and the meat area under the tracheobronchial clearance curve for the first 2.5 hours was 149 (28) units (range 99.3-187.3).

Figure 2 shows the nasal clearance plotted againsi the area under the tracheobronchial clearance curve for up to 2.5 hours after inhalation of radioaeroso The Spearman rank correlation test gave an $r_{s}$ value of $-0.79(\mathrm{p}<0.01)$.

Figure 3 shows the nasal clearance plotted against the area under the tracheobronchial clearance curve. for up to 6 hours after radioaerosol inhalation. The Spearman rank correlation test gave an $r_{s}$ value of $-0.94(\mathrm{p}<0.001)$.

\section{Discussion}

Several methods have been used to evaluate nasaf mucus transport. Initial studies used direct visuak isation of various substances applied to the mucosato surface. ${ }^{1213}$ Subsequently three main forms of tech nique have been described: the saccharin test, ${ }^{14}$ th $\mathbb{8}$ use of radiolabelled markers, and the use of radio opaque markers. Radiolabelled markers have bee used as microdroplets ${ }^{15}$ or single particles ${ }^{1617}$ with 


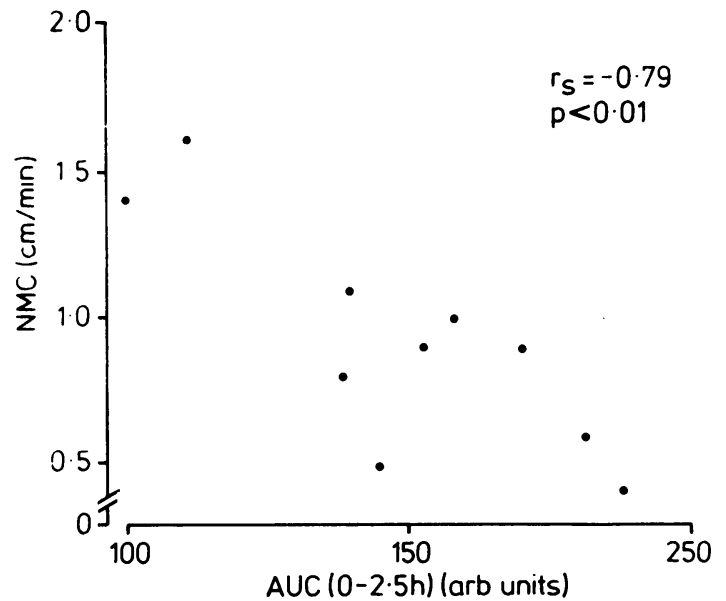

Fig 2 Nasal mucocilliary clearance (NMC) related to the area under the tracheobronchial clearance curve $(A U C$-in arbitrary units) in the first $2 \cdot 5$ hours: this represents clearance from the proximal airways.

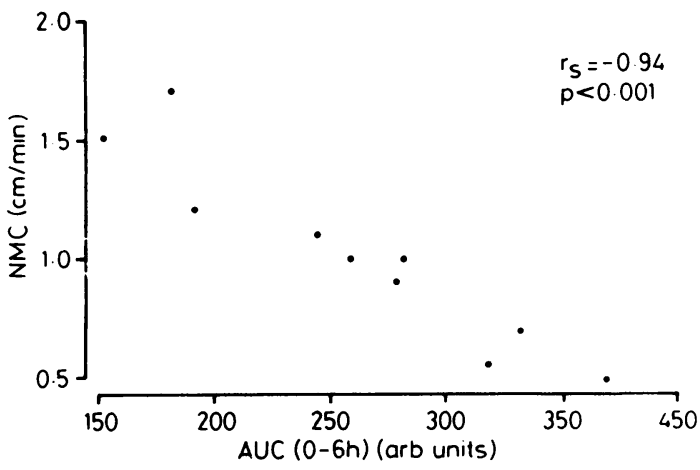

Fig 3 Nasal clearance $(N M C)$ related to the area under the tracheobronchial clearance curve ( $A U C$-in arbitrary units) in the first 6 hours: this represents clearance from most of the ciliated airways.

various types of detector. More recently a technique following the motion of radio-opaque discs with a fluoroscopic image intensifier ${ }^{18}$ has been used. These techniques have been compared by several groups, with varying results. ${ }^{1418-20}$ Comparisons of nasal and tracheobronchial mucociliary clearance have shown in one study an inverse correlation ${ }^{5}$ and in another study a weak association between tracheobronchial and nasal clearance as studied with a saccharin particle, ${ }^{4}$ and no correlation when nasal clearance was measured by single radiolabelled particles. ${ }^{4}$ In all these studies both clearance measurements were made on the same day. Our measurements of nasal and tracheobronchial clearance were made on different days, but we believe that this strengthens the relationship between our measurements. We could not measure both lung mucociliary clearance and nasal mucociliary clearance on the same day since radioactivity from one procedure might have interfered with measuring radioactivity from the other. As the reproducibility of the technique is within $\pm 11 \%$, measurements made on the same day would not significantly alter our findings.

Another explanation for the discrepancies observed is that the techniques used for measurement may differ widely. All these studies have used a radioaerosol technique for measuring lung tracheobronchial clearance. The nasal clearance test with saccharin used a water soluble substance, which dissolves in the mucus and periciliary layer and so alters the way in which it is cleared from the nose. Previous radiolabelled particle techniques for studying nasal clearance used a single particle, of various sizes up to $600 \mu \mathrm{m}$, whereas radioaerosols used in the lungs are under $10 \mu \mathrm{m}$. We set out to measure tracheobronchial and nasal clearance with particles of similar size. For both clearance measurements we used small particles $-5 \mu \mathrm{m}$ polystyrene (equivalent to $5 \mu \mathrm{m}$ unit density) for the lung and $2 \mu \mathrm{m}$ Teflon (equivalent to $3.2 \mu \mathrm{m}$ unit density) for the nose; they were in the form of an aerosol in the lung and a suspension in the nose. In the case of nasal clearance testing, it is difficult to assess how much localised retention of the radioactive material will depend on its initial distribution within the nose. For this reason we believe that the rate of movement of a visualised bolus of radioactivity offers the best indicator of nasal mucociliary function. We also measured tracheobronchial clearance by the area contained under the tracheobronchial clearance curve, which is inversely proportional to the clearance rate. We consider that this gives the best overall indication of the tracheobronchial clearance rate and is more comparable with the nasal clearance technique than using the time for a fixed percentage clearance, as in other studies. ${ }^{45}$

The closer relationship of overall tracheobronchial clearance (measured for the first 6 hours) than more proximal clearance (measured for the first 2.5 hours) to nasal clearance was surprising. Ciliary beat frequency in normal epithelium is similar to that of the proximal ciliated airways, but differs from that found in subsegmental bronchi. ${ }^{21}$ Ciliary beat frequency is only one element of mucociliary clearance. Other factors are the differences between the nasal mucosa and the tracheobronchial tree in terms of nerve and blood supply and mucus production. The thickness of the nasal mucosa can alter by $2-3 \mathrm{~mm}$ with ordinary climatic changes, whereas the bronchial mucosa rarely exceeds $1 \mathrm{~mm}$ in the presence of inflammation. ${ }^{3}$ This 
is due largely to the extremely rich vascular supply of the nasal mucosa. ${ }^{22}$ Differences between the nasal and bronchial mucosa in the response to environmental influences have been described in terms of the size and number of goblet cells and mucus glands. ${ }^{23}$ The innervation of the bronchial tree continues to be elucidated. Some differences between the response and the presence of a cholinergic, an adrenergic, and a non-cholinergic, non-adrenergic component in the nerve supply of the bronchial ${ }^{24}$ and nasal mucosa ${ }^{25}$ have been determined. A combination of these factors influences mucus clearance rate in both the nasal and the tracheobronchial passages.

Our results for nasal clearance are similar to those previously reported, and show a wide normal range. ${ }^{4-613-19}$ We have shown a close correlation between tracheobronchial clearance and nasal clearance in normal subjects, indicating that those factors that influence a particular individual's tracheobronchial clearance seem to exert the same influence on that individual's nasal clearance. As mucociliary clearance is such a highly developed and widespread mechanism for defence in the man this is not surprising. We conclude that using this technique nasal clearance is a useful guide to tracheobronchial clearance in healthy non-smoking subjects. It could be used as a screening procedure for congenital abnormalities of mucociliary clearance and for detecting those at particular risk from industrial exposure to inhaled carcinogens, such as in the woodworking industry. ${ }^{6}$ Further studies may show that abnormalities of nasal clearance are important to diseases of the lung. ${ }^{26} 27$

\section{References}

1 Proctor DF. The upper airways. 1-Nasal physiology and defense of the lungs. Am Rev Respir Dis 1977;115:97-235.

2 Proctor DR, Anderson I, Lundqvist G. Nasal mucociliary function in humans. In: Brain JD, Proctor DF, Reid LM, eds. Respiratory defence mechanisms. Vol 5, part 1. New York: M Dekker, 1977:427-52.

3 Proctor DF, Swift DL. The nose-a defence against the atmospheric environment. In: Davies CN, ed. Inhaled particles and vapours III. New York: Pergamon Press, 1971:59-69.

4 Anderson I, Camner P, Jensen PL, Philipson PL, Proctor DF. A comparison of nasal and tracheobronchial clearance. Arch Environ Health 1974;29:290-3.

5 Puchelle E, Aug F, Zahm JM, Bertrand A. A comparison of nasal and bronchial mucociliary clearance in young non-smokers. Clin Sci 1982;62:13-6.

6 Black A, Evans JC, Hadfield EH, Macbeth RG, Morgan A, Walsh M. Impairment of nasal mucociliary clearance in woodworkers in the furniture industry. $\mathrm{Br} J$ Ind Med 1974;31:10-7.

7 May KR. An improved spinning top homogeneous spray apparatus. J Appl Phys 1949;20:932-8.

8 Camner D, Philipson K, Svedburg J. Production of $7 \mu \mathrm{n}$ monodispersed fluorocarbon resin particles tagge with 18F. Int J Appl Radiat Isot 1971;22:349-53.

9 Pavia D, Sutton PP, Agnew JE, Lopez-Vidriero MT Newman SP, Clarke SW. Measurements of bronchia mucociliary clearance. Eur J Respir Dis 1983;64 (supp\$ 127):41-56.

10 Agnew JE, Bateman JRM, Watts M, Paramananda Pavia D, Clarke SW. The importance of aerosol pene $\vec{\omega}$ tration for lung mucociliary clearance studies. Ches? 1981;80 (suppl):843-6.

11 Cotes JE. Lung function: principles and application in medicine. 3rd ed. Oxford: Blackwell. Scientific Publica $\overrightarrow{\text {. }}$ tions, 1975.

12 Hilding A. Ciliary activity and cause of secretion cur rents of the nose. Mayo Clin Proc 1931;6:285-7.

13 Tremble GE. Clinical observations on the movement of nasal cilia. An experimental study. Laryngoscope 1948;58:206-24.

14 Anderson I, Camner P, Jensen PL, Philipson K, Procto DF. Nasal clearance in monozygotic twins. Am Re
Respir Dis 1974;110:301-5.

15 Proctor D, Wagner H. Clearance of particles from the human nose. Arch Environ Health 1965;11:366-71.

16 Quinlan MF, Salman SD, Swift DL, Wagner HN jun; Proctor DF. Measurement of mucociliary function in man. Am Rev Respir Dis 1969;99:13-23.

17 Anderson I, Lundqvist GR, Proctor DF. Human nasa mucosal function in a controlled climate. Arch Enviroof Health 1971;23:408-20.

18 Yergin B, Sakethoo K, Michaelson EO, Serafini SM Kovitz K, Sackner M. A roentgenographic method fo巳 measuring mucous velocity. J Appl Physio $\vec{t}$ 1978;44:964-8.

19 Puchelle E, Aug F, Pham QT, Bertrand A. Comparison of three methods for measuring nasal mucociliary clearance in man. Acta Otolaryngol 1981;91:297-303을

20 Brondeel L, Sonstabo R, Clement P, Van Kyckegheñ W, Van den Broek M. Value of the Tc99m particle tesī and the saccharin test in mucociliary examinations? Rhinology 1983;21:135-42.

21 Rutland J, Griffin WM, Cole PJ. Human ciliary bea frequency in epithelium from intrathoracic and extrathoracic airways. Am Rev Respir Dis 1982;125:100-5?

22 Andrews PM. A scanning electron microscopic study of the extrapulmonary respiratory tract. Am $J$ AnaE.

1974;139:399.
23 Burton PA, Dixon MC. A comparison of changes in mucus glands and goblet cells of nasal, sinus and bron chial mucosa. Thorax 1969;24:180-5.

24 Laitinen A, Portanen M, Hervonen A, Pelto-Huikko M Laitinen LA. VIP-like immunoreactive nerves in human respiratory tract. Light and electrone microscopic study. Histochemistry 1985;82:313-9.

25 Uddman R, Malm L, Sundler F. Peptide containing nerves in the nasal mucosa. Rhinology 1981;19:75-9.

26 Irwin RS, Carrao WM, Pratter MR. Chronic persisten cough in the adult: the sputum and frequency of causes and successful outcome of specific therapy. Am Re $\vec{\square}$ Respir Dis 1979;119:413-7.

27 Phipantankul CS, Slavin RG. Bronchial asthma pros duced by paranasal sinusitis. Arch Otolaryngof 1974;100:109. 